\title{
Usefulness of limited sampling strategy for mycophenolic acid area under the curve considering postoperative days in living- donor renal transplant recipients with concomitant prolonged-release tacrolimus
}

Tomoyuki Enokiya', Kouhei Nishikawa², Yuichi Muraki', Takuya Iwamoto' ${ }^{1}$, Hideki Kanda², Yoshiki Sugimura² and Masahiro Okuda ${ }^{*}$

\begin{abstract}
Background: The optimal dose of mycophenolate mofetil (MMF) in renal transplant patients has been recommended to be decided on the basis of area under the concentration-time curve ( $\mathrm{UUC}_{0-12}$ ) of mycophenolic acid (MPA). Although meta-analysis has revealed that postoperative day (POD) is an influencing factor in MPA pharmacokinetics, there are no reports regarding a limited sampling strategy (LSS) for MPA AUC in consideration of POD. The aim of this study was to construct of an LSS considering POD that appropriately expresses the MPA AUC following renal transplantation and evaluation of the usefulness.

Methods: Serum concentration-time profiles (measured $\mathrm{AUC}_{0-12}$ ) comprising nine sampling points over $12 \mathrm{~h}$ were analyzed in 36 living-donor renal transplant recipients after MMF administration with concomitant once-daily prolonged-release tacrolimus. Two LSSs were developed by stepwise multiple regression analysis (Method A: not classified by PODs; Method B: classified by PODs into POD $<31$ and POD $\geq 31$ ). Each LSS comprised four blood-sampling points within $6 \mathrm{~h}$ after MMF administration. Precision and reliability were verified by using root-mean-square error (RMSE), correlation coefficient $\left(R^{2}\right)$, and coefficient of determination $\left(q^{2}\right)$ by using leave-one-out cross-validation. The absolute values of the difference between measured and estimated AUCs (delta AUC) were compared for both estimating equations.

Results: One-hundred samples obtained from 36 recipients for $\mathrm{AUC}_{0-12}$ comprised $\mathrm{POD}<31(n=39)$ and $P O D \geq 31(n=61)$. Estimation of $A \cup C_{0-12}$ by Method B resulted in better accuracy and reliability (Method A: $R M S E=5.5, R^{2}=0.85, q^{2}=0.83 ;$ Method $B: P O D<31: R M S E=5.5, R^{2}=0.86, q^{2}=0.83 ; P O D \geq 31: R M S E=3.9, R^{2}=0.92$, $\left.\mathrm{q}^{2}=0.89\right)$ and significantly lower median delta AUC compared with that by Method A (delta AUC: $2.6(0.0-11.6)$ v.s. 3.9 $(0.1-18.1), p=0.032)$.
\end{abstract}

Conclusion: These results suggest that LSS, classified as $\mathrm{POD}<31$ or POD $>31$, would provide more accurate and reliable estimation of MPA $\mathrm{AUC}_{0-12}$ in Japanese living-donor renal transplant patients.

Keywords: Mycophenolate mofetil, Limited sampling strategy, Living-donor renal transplantation, Mycophenolic acid, Therapeutic drug monitoring

\footnotetext{
* Correspondence: okudam@clin.medic.mie-u.ac.jp

'Department of Pharmacy, Mie University Hospital, Faculty of Medicine, Mie

University, 2-174 Edobashi, Tsu, Mie 514-8507, Japan

Full list of author information is available at the end of the article
} 


\section{Background}

Mycophenolate mofetil (MMF), a prodrug of the immunosuppressant mycophenolic acid (MPA), has been widely used for the prevention of rejection in solid organ transplant patients [1-3]. MMF is administered to patients who have undergone renal transplantation at a dosage of $0.5-1.5 \mathrm{~g}$ twice daily. After oral administration, MMF is rapidly absorbed and hydrolyzed to MPA [4], and is then inactivated to MPA glucuronide by UDP-glucuronosyltransferase [5].

Numerous studies have demonstrated the relationship between area under the concentration-time curve $\left(\mathrm{AUC}_{0-12}\right)$ of MPA and both risk of rejection [4, 6-12] and hematologic side effects $[11,13]$. A target range of 30-60 $\mathrm{mg} \mathrm{h} / \mathrm{L}$ for the MPA $\mathrm{AUC}_{0-12}$ has been proposed as a guide to MMF dosage in renal transplant patients in these studies. There were large inter- and intraindividual variations in MPA $\mathrm{AUC}_{0-12}$ [13]. However, routine measurement of full MPA $\mathrm{AUC}_{0-12}$ for $12-\mathrm{h}$ dose intervals is cumbersome and cost-prohibitive. Limited sampling strategies (LSSs) have been developed in several countries for estimating $\mathrm{MPA} \mathrm{AUC}_{0-12}$ to overcome these difficulties [14].

van Hest et al. [15] reported that MPA pharmacokinetics are affected by the patient's renal function, serum albumin concentration, and dosage of immunosuppressants, including calcineurin inhibitors that are dependent on the post-renal transplant period. Moreover, several studies reported that oral MPA clearance is inversely proportional to postoperative days (POD), achieving gradual stability [7, 11]. Therefore, sampling points to estimate MPA $\mathrm{AUC}_{0-12}$ might vary according to POD. However, there is no report demonstrating LSS design with consideration of POD. The aim of this study was to develop LSS with consideration of POD, and to evaluation of the usefulness of these LSSs in Japanese renal transplant patients.

\section{Methods}

\section{Patients}

This study was performed on all 36 patients who underwent living-donor renal transplantation at Mie University Hospital between November 2005 and August 2015. One-hundred serum MPA concentration-time profiles were prospectively obtained between November 2012 and September 2015.

\section{Data collection}

Demographic data including concomitant drug use were obtained by reviewing electronic medical records of the patients. Concomitant drugs that are documented in Lexicomp, integrated with UpToDate (version 2014; Wolters Kluwer Health, Philadelphia, PA, USA) were considered.

\section{Assay of serum MPA concentration}

Serum was separated by centrifugation at $1700 \times g$ for 10 min by using serum separation tubes. Serum MPA concentration was determined by using a homogeneous particle-enhanced turbidimetric inhibition immunoassay (PETINIA) technique on a DIMENSION ${ }^{\bullet}$ Xpand Plus Integrated Chemistry System (Siemens Healthcare Diagnostics K.K., Tokyo, Japan).

\section{Immunosuppression regimen}

All patients received a basic immunosuppression regimen of MMF (CellCept; Chugai Pharmaceutical Co., Ltd., Tokyo, Japan), once-daily prolonged-release tacrolimus (Graceptor; Astellas, Tokyo, Japan,), methylprednisolone, and basiliximab (Simulect i.v. injection; Novartis Pharmaceuticals, Tokyo, Japan). In addition, patients with blood type incompatibility received rituximab at a dose of $200 \mathrm{mg}$ on preoperative day 4. The MMF was taken on preoperative day 4 at a fixed starting dose of $0.5 \mathrm{~g}$ twice daily, $1 \mathrm{~g}$ twice daily from POD $0,0.75 \mathrm{~g}$ twice daily from POD 15, and $0.5 \mathrm{~g}$ twice daily from POD 60. The starting dose of tacrolimus was $0.1 \mathrm{mg} / \mathrm{kg} /$ day, adjusted based on whole-blood concentration (target concentration: 6.0-8.0 ng/mL). Methylprednisolone was started at a dose of $20 \mathrm{mg} /$ day and administered at a dose of $250 \mathrm{mg}$ during surgery, reduced gradually to the maintenance dose of $4 \mathrm{mg} /$ day from POD 30. Basiliximab was injected intravenously at a dose of $20 \mathrm{mg}$ within $2 \mathrm{~h}$ before the operation and on POD 4 .

\section{Determination of MPA AUC $_{0-12}$}

Serum MPA concentration was determined just before dosage and at $0.5,1,2,3,4,6,8$, and $12 \mathrm{~h}$ after administration. The MPA AUC $0-12$ was calculated using a linear trapezoidal rule. The $\mathrm{AUC}_{0-12}$ of MPA was determined on POD 7, POD 14, POD 21-28, and POD $\geq 31$ according to recommendation on the application of therapeutic drug monitoring to MMF therapy in transplantation [16].

\section{Development of a POD-based LSS}

One-hundred serum MPA concentration-time profiles were classified into four groups on the basis of POD (POD 7, POD 14, POD 21-28 and POD $\geq 31$ ). MPA clearance (oral MMF dose per $\mathrm{AUC}_{0-12}$ ) was compared between these four groups by using multiple comparison test. POD-based LSSs were developed using multiple comparison analysis. Each estimating equation was developed by using stepwise multiple regression analysis, and comprised four blood-sampling points over $6 \mathrm{~h}$ after drug administration.

\section{Evaluation of estimating equations}

Precision was evaluated by using Spearman's rank correlation test, root-mean-square error (RMSE), correlation 
coefficient $\left(\mathrm{R}^{2}\right)$ by least squares method, and correlation between measured AUC and estimated AUC. Reliability was evaluated by using coefficient of determination $\left(\mathrm{q}^{2}\right)$, calculated using leave-one-out cross-validation (LOOCV).

The absolute value of the difference (delta AUC) between measured and estimated AUCs was compared between each LSS by using the Wilcoxon matched pair test.

\section{Statistical analysis}

Spearman's rank correlation test, RMSE, and least squares method were performed using JMP ${ }^{\oplus}$ Ver. 7.0 (SAS Institute, Cary, NC, USA). Wilcoxon matched pair and Kruskal-Wallis tests were performed using GraphPad Prism Ver. 5.01 (GraphPad Software, Inc., San Diego, CA, USA). LOOCV and multiple comparisons by Wilcoxon signed-rank test (Holm's method) were performed using GNU R Ver. 3.1.0 for windows. A $P$ value $<0.05$ was considered significant.

\section{Results}

\section{Development of POD-based LSS}

The $\mathrm{AUCs}_{0-12}$ of MPA on POD $7(n=13)$, POD 14 $(n=16)$, POD 21-28 $(n=10)$, and POD $\geq 31(n=61)$ were determined as described in the materials and methods section. Significant differences in the oral clearance of MPA were found between POD $\geq 31$ and POD 7 or POD 14 by multiple comparison analysis (Fig. 1). Moreover, MPA oral clearance tended to be lower in POD $\geq 31$ compared with that in POD 21-28. There were no significant differences in MPA oral clearance between POD 7, POD 14, and POD 21-28. On the basis of these results, the LSSs for PODs $<31$ or $\geq 31$ were defined by using Method B.

\section{Demographic data}

Table 1 displays the data on patient characteristics including gender, primary disease, age, body weight, creatinine

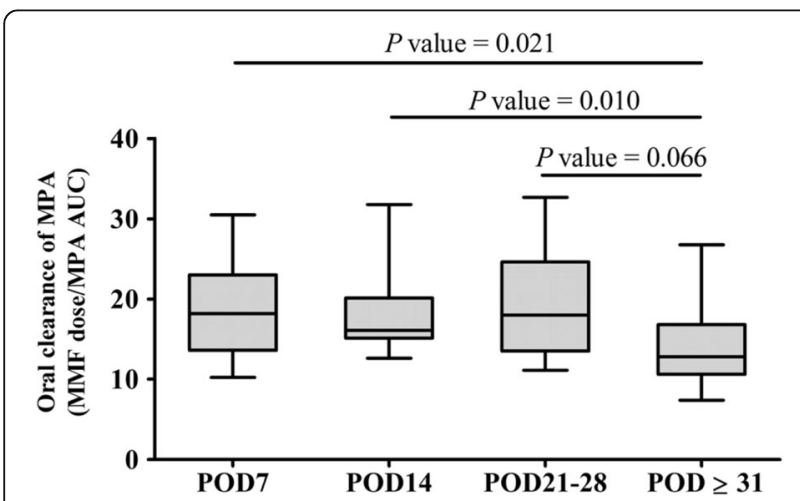

Fig. 1 Differences in clearance of mycophenolic acid in postoperative periods. Graphs display median and interquartile range. AUC: area under the concentration-time curve; POD: postoperative day. Number of actual $\mathrm{AUC}_{0-12}$ of mycophenolic acid: 13 (POD 7), 16 (POD 14), 10 (POD 21-28), 61 (POD $\geq 31$ )
Table 1 Demographic data in living donor renal transplant recipients

\begin{tabular}{|c|c|}
\hline & $\begin{array}{l}\text { The number of patients (\%) or } \\
\text { median [minimum-maximum] }\end{array}$ \\
\hline Male & $17(47.2)$ \\
\hline \multicolumn{2}{|l|}{ Primary disease } \\
\hline diabetic nephropathy & $9(25.0)$ \\
\hline IgA nephropathy & $7(19.4)$ \\
\hline polycystic kidney & $3(8.3)$ \\
\hline chronic glomerulonephritis & $2(5.5)$ \\
\hline focal glomerulosclerosis & $2(5.5)$ \\
\hline Alport syndrome & $1(2.7)$ \\
\hline cystinosis & $1(2.7)$ \\
\hline mesangial proliferative glomerulonephritis & $1(2.7)$ \\
\hline unknown & $10(26.0)$ \\
\hline $\mathrm{Age}^{\mathrm{a}}$ & $47[28-66]$ \\
\hline Body weight $(\mathrm{kg})^{\mathrm{a}}$ & $55.5[34.8-105.9]$ \\
\hline Serum albumin $(g / d L)^{a}$ & $4.0[2.9-5.1]$ \\
\hline Serum creatinine $(\mathrm{mg} / \mathrm{dL})^{\mathrm{a}}$ & $1.1[0.5-2.9]$ \\
\hline Estimated creatinine clearance $(\mathrm{mL} / \mathrm{min})^{\mathrm{a}}$ & $57.5[24.9-113.8]$ \\
\hline Total bilirubin $(\mathrm{mg} / \mathrm{dL})^{\mathrm{a}}$ & $0.6[0.2-1.6]$ \\
\hline Alanine aminotransferase $(\mathrm{IU} / \mathrm{mL})^{a}$ & $12[4-117]$ \\
\hline Aspartate aminotransferase $(\mathrm{IU} / \mathrm{mL})^{\mathrm{a}}$ & $17[6-109]$ \\
\hline \multicolumn{2}{|l|}{ Mycophenolate mofetil dose at one time (mg) } \\
\hline Postoperative day < 31 & $1000[500-1000]$ \\
\hline Postoperative day $\geq 31$ & $500[250-1000]$ \\
\hline \multicolumn{2}{|c|}{ Actual $A \cup C_{0-12}$ of mycophenolic acid $(\mu \mathrm{g} \cdot \mathrm{h} / \mathrm{mL})$} \\
\hline Postoperative day < 31 & $52.7[23.6-89.2]$ \\
\hline Postoperative day $\geq 31$ & $43.7[21.5-87.6]$ \\
\hline \multicolumn{2}{|l|}{ Oral clearance of mycophenolate acid } \\
\hline Postoperative day < 31 & $18.0[10.2-32.7]$ \\
\hline Postoperative day $\geq 31$ & $12.5[6.4-34.1]$ \\
\hline \multicolumn{2}{|l|}{ Postoperative day } \\
\hline Postoperative day $<31$ & $14[7-21]$ \\
\hline Postoperative day $\geq 31$ & 359 [34-2832] \\
\hline \multicolumn{2}{|l|}{ Concomitant drug $a, b$} \\
\hline \multicolumn{2}{|l|}{ Postoperative day $<31$} \\
\hline Proton pump inhibitor & $34(34.0)$ \\
\hline Proton pump inhibitor + Quinolone & $4(4.0)$ \\
\hline \multicolumn{2}{|l|}{ Postoperative day $\geq 31$} \\
\hline Quinolone & $22(22.0)$ \\
\hline Proton pump inhibitor & $16(16.0)$ \\
\hline Proton pump inhibitor + Quinolone & $17(17.0)$ \\
\hline Proton pump inhibitor + Valganciclovir & $2(2.0)$ \\
\hline
\end{tabular}

${ }^{a}$ These data were measured on the day the $\mathrm{AUC}_{0-12}$ was determined $(n=100)$ ${ }^{\mathrm{b}}$ Concomitant drugs that may influence MPA pharmacokinetics were examined by using Lexicomp $\odot$ integrated in UpToDate ${ }^{\ominus}$ (version 2014; Wolters Kluwer Health, Philadelphia, PA, USA) 
clearance estimated by Cockcroft and Gault formula, alanine aminotransferase, aspartate aminotransferase, total bilirubin, concomitant drugs, and measured MPA $\mathrm{AUC}_{0-}$ 12. Figure 2 shows the median concentration-time profile. Median (range) $\mathrm{C} 0, \mathrm{t}_{\max }$, and $\mathrm{C}_{\max }$ were $2.9(0.3-7.7) \mu \mathrm{g} /$ $\mathrm{mL}, 2.3(0.5-6.0) \mathrm{h}$, and $11.3(3.3-27.3) \mu \mathrm{g} / \mathrm{mL}$ for POD < 31, and $1.9(0.2-5.7) \mu \mathrm{g} / \mathrm{mL}, 2.2(0.5-6.0) \mathrm{h}$, and 11.9 (3.4-43.2) $\mu \mathrm{g} / \mathrm{mL}$ for POD $\geq 31$, respectively. Median concentrations of MPA for POD $\geq 31$ during 1 and $4 \mathrm{~h}$ after administration were mostly constant similarly as those for POD $<31$ (Fig. 2). In contrast, the $\mathrm{AUC}_{0-12}$ of MPA per dose $(\mathrm{mg})$ for POD $<31$ was significantly lower than that for $\mathrm{POD} \geq 31$ (median (range): 0.056 (0.031-0.098) v.s. 0.078 (0.029-0.155), $P<0.0001)$.

\section{Evaluation of estimating equations}

The $P$ value, RMSE, $\mathrm{R}^{2}$, and $\mathrm{q}^{2}$ of each estimating equation are shown in Table 2 . The $P$ values of all estimating equations were less than 0.001 . The worst precision (RMSE and $R^{2}$ ) and reliability $\left(q^{2}\right)$ were observed in MPA $\mathrm{AUC}_{0-12}$ estimation of POD $<31$ by Method A. Estimation by Method $\mathrm{B}$ resulted in better precision (RMSE and $\mathrm{R}^{2}$ ) and reliability $\left(\mathrm{q}^{2}\right)$ than that by Method A. The correlation of Method B with estimated and measured AUC was better than that of Method A, as demonstrated by the results where corresponding slope, intercept, and $R^{2}$ values of Method B were much closer to 1,0 , and 1 , respectively (Fig. 3). Wilcoxon matched pair test indicated that delta AUC estimated by Method B was significantly lower than that by Method A (Fig. 4).

\section{Discussion}

In this study, two different approaches to developing LSSs for the estimation of $\mathrm{AUC}_{0-12}$ were evaluated by statistical analyses (Method A: not classified by POD,
Method B: classified by POD into $<31$ or $\geq 31$ based on difference in MPA systemic clearance). Precision (RMSE and $\left.R^{2}\right)$ and reliability $\left(q^{2}\right)$ were compared between Method A and Method B, and Method B provided better estimation of $\mathrm{AUC}_{0-12}$ compared with Method A. Moreover, delta AUC of Method B was lower than that of Method A. Therefore, these results suggested that LSSs considering POD would provide more precise and reliable estimation of MPA $\mathrm{AUC}_{0-12}$.

It has been reported that patients within 1 month post-transplant have lower MPA $\mathrm{AUC}_{0-12}$ than patients between 3 and 6 months post-transplant $[7,11]$. Moreover, van Hest et al. [15] reported that POD was a significant factor affecting the pharmacokinetics of MPA. In our present study, the results of multiple comparisons of MPA oral clearance between four groups classified by POD proved that MPA oral clearance on POD $<31$ was higher than that of $P O D \geq 31$ (Fig. 1). Furthermore, estimated $\mathrm{AUC}_{0-12}$ on $\mathrm{POD}<31$ did not exhibit better precision and reliability than that on $\mathrm{POD} \geq 31$ when Method A was applied (RMSE, $\mathrm{R}^{2}$, and $\mathrm{q}^{2}$ for POD $<31$ or $\mathrm{POD} \geq 31$ were $6.7,0.78$, and 0.73 or $4.2,0.90$, and 0.89 , respectively (data not shown)). We also compared MPA clearance between POD $<91$ and $P O D \geq 91$. MPA clearance was lower in POD $<91$ compared with that in $\mathrm{POD} \geq 91$ (17.2 [8.6-32.7] v.s. 12.5 [6.4-34.1], $P=0.0009$ ). However, there was no significant difference in MPA clearance between POD31-90 and POD $\geq 91$ (POD31-90: 14.3 [8.6-19.8] v.s. $\mathrm{POD} \geq 91: 12.5$ [6.4-34.1], $p=0.16$ ). Moreover, LSSs classified by PODs into POD $<91$ and POD $\geq$ 91 was not better than Method $\mathrm{B}\left(\mathrm{POD}<91: \mathrm{R}^{2}=0.86\right.$, $\mathrm{RMSE}=5.86, \mathrm{q}^{2}=0.82 ; \mathrm{POD} \geq 91: \mathrm{R}^{2}=0.91, \mathrm{RMSE}=3.54$, $\mathrm{q}^{2}=0.88$ ). Therefore, data from both these previous studies and our study support our opinion that estimation equations for MPA $\mathrm{AUC}_{0-12}$ with built-in consideration of

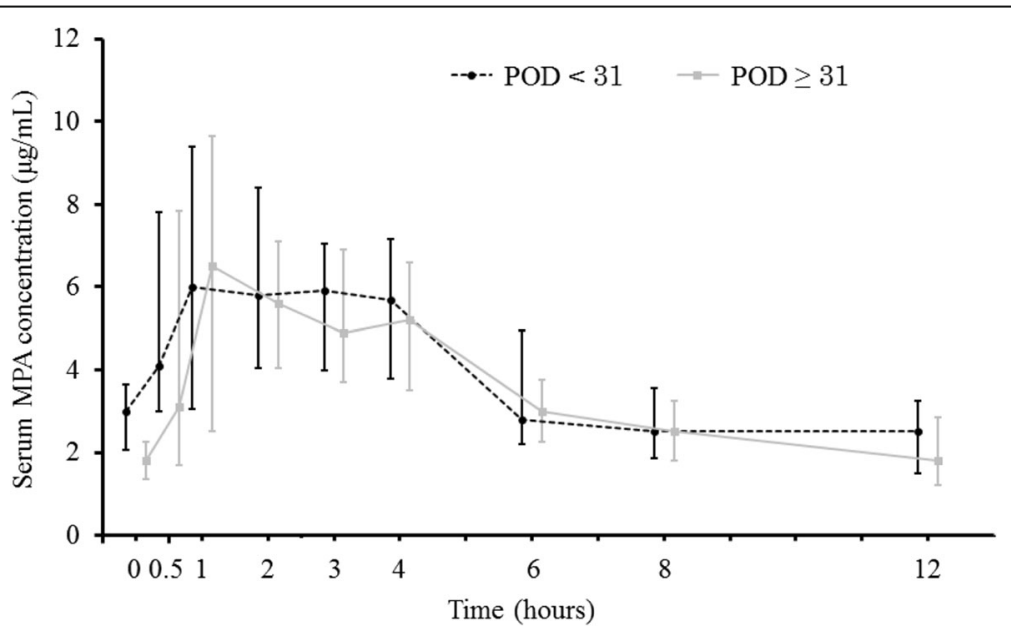

Fig. 2 Median (interquartile range) serum concentration-time profiles of MPA. Block circle points on broken line depict POD $<31$ and gray square points on solid line depict POD $\geq 31$ MPA: mycophenolic acid; POD: postoperative day 
Table 2 Correlation with measured $\mathrm{AUC}_{0-12}$, accuracy, and reliability of each estimated formula

\begin{tabular}{|c|c|c|c|c|c|c|c|}
\hline & & $n$ & Equations for $\mathrm{AUC}_{0-12}$ estimation & $P$ value & RMSE $^{* *}$ & $R^{2 \#}$ & $q^{2 \# \#}$ \\
\hline Method A & All patients & 100 & $7.4+2.3 \times C_{0 h}+1.2 \times C_{1 h}+2.3 \times C_{3 h}+4.4 \times C_{6 h}$ & $<0.0001$ & 5.5 & 0.85 & 0.83 \\
\hline \multirow[t]{2}{*}{ Method B } & $\mathrm{POD}<31$ & 39 & $10.6+1.1 \times C_{1 h}+1.1 \times C_{2 h}+2.0 \times C_{4 h}+3.9 \times C_{6 h}$ & $<0.0001$ & 5.5 & 0.86 & 0.83 \\
\hline & $\mathrm{POD} \geq 31$ & 61 & $3.8+3.5 \times C_{0 h}+1.2 \times C_{1 h}+1.9 \times C_{3 h}+5.4 \times C_{6 h}$ & $<0.0001$ & 3.9 & 0.92 & 0.89 \\
\hline
\end{tabular}

$C_{\text {time }}$ serum mycophenolic acid concentration at time after administration, $P O D$ postoperative day

${ }^{*} P$ value: Spearman's rank correlation test, ${ }^{* *} R M S E$ root-mean-square error, ${ }^{\#} R^{2}$ least squares method, ${ }^{\# \#} q^{2}$ leave-one-out cross-validation

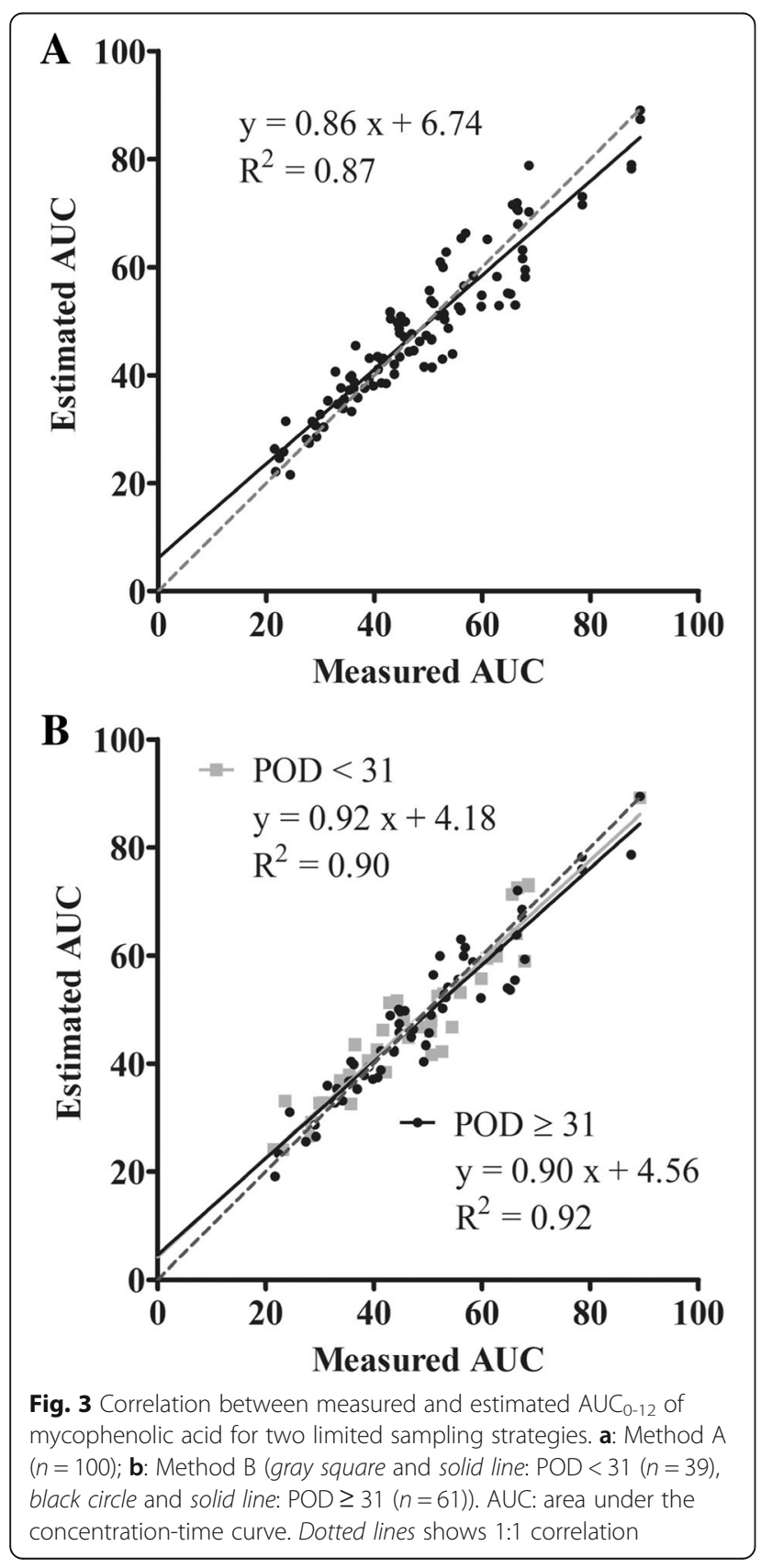

POD should have better precision and reliability. This is because there is a difference in MPA pharmacokinetics between $\mathrm{POD}<31$ and $\mathrm{POD} \geq 31$.

This study has some limitations. First, measured serum MPA concentration includes serum acyl-glucuronide metabolite (AcMPAG) concentration because serum MPA concentration was measured by PETINIA, and the antibody used in PETINIA cross-reacts with AcMPAG [16]. Therefore, the universality of LLS developed in the present study may be limited. Second, the contribution of concomitant drugs is not completely overseen by the present study. However, in LSS by Method B, we performed multiple comparison testing of delta AUC between groups classified by concomitant drug usage, proving there is no difference in delta AUC between groups (Additional files 1,2 , and 3). Therefore, LSSs devised in this study seem to be slightly influenced by the concomitant use of drugs such as quinolone, proton pump inhibitors, and valganciclovir. Third, we cannot explain the mechanism by which mycophenolate clearance changed according to POD.




Because of small number of patients involved in the present study, some MPA concentration profiles has been obtained from same patients.

Pawinski et al. [17] reported that $\mathrm{AUC}_{0-12}$ estimation comprising three blood-sampling points $(0,0.5$, and $2.0 \mathrm{~h}$ ) may provide good prediction of MPA $\mathrm{AUC}_{0-12}$ in renal transplant patients receiving concomitant tacrolimus. However, we could not find literature on LSS that satisfied the following conditions: i) concomitant use of tacrolimus as a calcineurin inhibitor, ii) containing data within 1 month after renal transplantation, and iii) determination of MPA using the PETINIA method. We evaluated the LSS developed by Pawinski et al., which had been well analyzed and meets two conditions (concomitant with tacrolimus and containing data within 1 month after renal transplantation). However, using this equation, we could not obtain a good correlation between the estimated and measured MPA AUCs in our study population $\left(y=0.74 x+8.32, R^{2}=0.47\right.$, Additional file 4$)$. The reason for this might be explained as follows: i) patients in our study were concomitantly administered once daily prolonged release tacrolimus; ii) our study population included many early post-transplant patients; and iii) the frequency of UGT1A9 (a metabolic enzyme of MPA) variants (UGT1A9*1, UGT1A9"1c, and UGT1A9*3) varies in the Caucasian, African, and Asian populations [18].

It has been reported that the MPA $\mathrm{AUC}_{0-12}$, measured by PETINIA method, are overestimated in comparison with MPA concentrations measured by high performance liquid chromatograph (HPLC) method [19]. Miura et al. compared LSS on the POD28 and 1 year after transplantation in Japanese kidney transplant patients. In that study, the values of MPA $\mathrm{AUC}_{0-12}$ (mean [standard deviation (SD)]) measured by HPLC method, were 63.9 [28.9] on the POD 28 and 58.1 [24.3] on 1 year after transplantation, respectively [20,21]. On the other hand, in the present study, the values of MPA $\mathrm{AUC}_{0-12}$ (mean [SD]) were 44.6 [14.4] on the POD 21-28 $(n=10)$ and 45.2 [10.2] on about 1 year after transplantation (POD 345-401), respectively (data not shown). Although dose of MMF in our study was similar to that in their study, the mean of MPA $\mathrm{AUC}_{0-12}$ in our study population were lower than that by Miura et al.. In our study, patients was administered prolonged-release tacrolimus concomitantly, whereas in the study of Miura et al., tacrolimus administered to patients was not prolonged-release formulation. Although controversy remains about the interaction between MMF and tacrolimus, prolongedrelease tacrolimus formulation might less effect on pharmacokinetics of MPA, since the formulation decreases $\mathrm{C}_{\max }$ of tacrolimus. Therefore, the difference in formulation of tacrolimus might be the reason for the difference of the MPA $\mathrm{AUC}_{0-12}$ between study of Miura et al. and our present study.
Yamaguchi et al. [22] reported the estimation equations for MPA $\mathrm{AUC}_{0-12}$ in the Japanese population. However, the usefulness of the estimation equation developed by Yamaguchi et al. was limited because the correlation between the measured and estimated $\mathrm{AUC}_{0-12}$ at 1 and 3 months after renal transplantation was not good enough and cross validation of the estimation equation was not performed. In this study, it was demonstrated that our estimation equation was better correlation than that of Yamaguchi et al. and we validated its reliability by cross validation.

In the present study, LSS consisting of four timed samples within $6 \mathrm{~h}$ after administration provided accurate and reliable estimation of MPA $\mathrm{AUC}_{0-12}$ and was best among the verified estimation equations (Additional file 5). It is known that MPA plasma concentration profile shows a secondary peak at around $6 \mathrm{~h}$ after administration because of enterohepatic circulation [16] and its mean contribution to the overall MPA $\mathrm{AUC}_{0-12}$ is $37 \%$ (10-61\%) [23]. Therefore, MPA blood concentration at around $6 \mathrm{~h}$ after administration should be important for estimating MPA AUC $0-12$. Measurement of MPA $\mathrm{AUC}_{0-12}$ by using 10-12 timed blood samples is a burden on both the patient and medical staff, requiring laboratory resources, considerable quantities of patients' blood, and a minimum $12 \mathrm{~h}$ stay in hospital. Therefore, LSSs that developed by this study, not only Method B but also Method A, will reduce the burden on both patients and medical staff and measurement costs.

\section{Conclusions}

This study suggested that LSSs with consideration of POD provide more accurate and reliable estimations of MPA $\mathrm{AUC}_{0-12}$ in Japanese renal transplant patients receiving concomitant tacrolimus therapy.

\section{Additional files}

\section{Additional file 1: Multiple comparison of delta $\mathrm{AUC}_{0-12}$ between five groups classified according to concomitant drug usage (all patients). (PPTX $61 \mathrm{~kb}$ ) \\ Additional file 2: Multiple comparison of delta $\mathrm{AUC}_{0-12}$ between three groups classified according to concomitant drug usage (POD<31). (PPTX 53 kb) \\ Additional file 3: Multiple comparison of delta $\mathrm{AUC}_{0-12}$ between five groups classified according to concomitant drug usage (POD $\geq 31$ ). (PPTX $56 \mathrm{~kb}$ ) \\ Additional file 4: Correlation between measured and estimated $\mathrm{AUC}_{0-12}$ estimated by using Pawinski's estimation formula. (PPTX 56 kb) \\ Additional file 5: Equations obtained using limited sampling time points for the prediction of MPA AUC ${ }_{0-12}$, Correlations with measured MPA AUC $\mathrm{C}_{0-12}$ and RMSE of estimated $\mathrm{AUC}_{0-12}$ (XLSX $\left.11 \mathrm{~kb}\right)$}

\section{Abbreviations}

AcMPAG: Acyl-glucuronide metabolite; AUC: Area under the concentrationtime curve; LOOCV: Leave-one-out cross-validation; LSSs: Limited sampling strategies; MMF: Mycophenolate mofetil; MPA: Mycophenolic acid; 
PETINIA: Particle-enhanced turbidimetric inhibition immunoassay; POD: Postoperative days; RMSE: Root-mean-square error

\section{Acknowledgements}

Not applicable.

\section{Funding}

There are no funding sources for this report.

\section{Availability of data and materials}

All data generated or analyzed during this study are included in this published article and its additional files.

\section{Authors' contributions}

$\mathrm{TE}, \mathrm{KN}, \mathrm{TI}$ and $\mathrm{MO}$ conceived and designed this study. YM, HK, YS and MO supervised the conduct of this study and data collection. TE provided statistical advice on study design and analyzed the data. TE drafted the manuscript, and all authors contributed substantially to its revision. MO takes responsibility for this paper as a whole. All authors read and approved the final manuscript.

\section{Competing interest}

The authors declare that they have no competing interests.

\section{Consent for publication}

Not applicable.

\section{Ethics approval and consent to participate}

This study was conducted in accordance with the Declaration of Helsinki and its amendments, and was approved by the ethics committee of Mie University Graduate School of Medicine and Faculty of Medicine (Approval No.: 2505).

\section{Publisher's Note}

Springer Nature remains neutral with regard to jurisdictional claims in published maps and institutional affiliations.

\section{Author details}

${ }^{1}$ Department of Pharmacy, Mie University Hospital, Faculty of Medicine, Mie University, 2-174 Edobashi, Tsu, Mie 514-8507, Japan. Department of Nephro-Urologic Surgery and Andrology, Mie University Hospital, Mie University, Tsu, Mie 514-8507, Japan.

Received: 24 February 2017 Accepted: 18 June 2017

Published online: 24 June 2017

\section{References}

1. The European Mycophenolate Mofetil Cooperative Study Group. Placebocontrolled study of mycophenolate mofetil combined with cyclosporin and corticosteroids for prevention of acute rejection. Lancet. 1995;345:1321-5.

2. Kobashigawa J, Miller L, Renlund D, Mentzer R, Alderman E, Bourge R, et al. A randomized active-controlled trial of mycophenolate mofetil in heart transplant recipients. Transplantation. 1998:66:507-15.

3. Wiesner R, Rabkin J, Klintmalm G, McDiarmid S, Langnas A, Punch J, et al. A randomized double-blind comparative study of mycophenolate mofetil and azathioprine in combination with cyclosporine and corticosteroids in primary liver transplant recipients. Liver Transpl. 2001;7:442-50.

4. Shaw LM, Nicholls A, Hale M, Armstrong W, Oellerich M, Yatscoff R, et al. Therapeutic monitoring of mycophenolic acid. A consensus panel report. Clin Biochem. 1998;31:317-22.

5. Bullingham RE, Nicholls AJ, Kamm BR. Clinical pharmacokinetics of mycophenolate mofetil. Clin Pharmacokinet. 1998;34:429-55.

6. Takahashi K, Ochiai T, Uchida K, Yasumura T, Ishibashi M, Suzuki S, et al. Pilot study of mycophenolate mofetil (RS-61443) in the prevention of acute rejection following renal transplantation in Japanese patients. Transplant Proc. 1995:27:1421-4.

7. Hale MD, Nicholls AJ, Bullingham RE, Hené R, Hoitsma A, Squifflet JP, et al. The pharmacokinetic-pharmacodynamic relationship for mycophenolate mofetil in renal transplantation. Clin Pharmacol Ther. 1998;64:672-83.

8. Nichols AJ. Opportunities for therapeutic monitoring of mycophenolate mofetil dose in renal transplantation suggested by the pharmacokinetic/ pharmacodynamic relationship for mycophenolic acid and suppression of rejection. Clin Biochem. 1998:31:329-33.

9. van Gelder T, Hilbrands LB, Vanrenterghem Y, Weimar W, de Fijter JW, Squifflet JP, et al. A randomized double-blind, multicenter plasma concentration controlled study of the safety and efficacy of oral mycophenolate mofetil for the prevention of acute rejection after kidney transplantation. Transplantation. 1999;68:261-6.

10. DeNofrio D, Loh E, Kao A, Korecka M, Pickering FW, Craig KA, et al. Mycophenolic acid concentrations are associated with cardiac allograft rejection. J Heart Lung Transplant. 2000;19:1071-6.

11. Shaw LM, Korecka M, Aradhye S, Grossman R, Bayer L, Innes C, et al. Mycophenolic acid area under the curve values in African American and Caucasian renal transplant patients are comparable. J Clin Pharmacol. 2000;40:624-33.

12. Shaw LM, Korecka M, DeNofrio D, Brayman KL. Pharmacokinetic pharmacodynamic, and outcome investigations as the basis for mycophenolic acid therapeutic drug monitoring in renal transplant patients. Clin Biochem. 2001;34:17-22.

13. Mourad M, Malaise J, Eddour DC, Meyer MD, König J, Schepers R, et al. Correlation of mycophenolic acid pharmacokinetic parameters with side effects in kidney transplant patients treated with mycophenolate mofetil. Clin Chem. 2001:47:88-94.

14. Tett SE, Saint-Marcoux F, Staatz CE, Brunet M, Vinks AA, Miura M, et al. Mycophenolate, clinical pharmacokinetics, formulations, and methods for assessing drug exposure. Transplant Rev (Orlando). 2011;25:47-57.

15. van Hest R, Mathot R, Vulto A, Weimar W, van Gelder T. Predicting the usefulness of therapeutic drug monitoring of mycophenolic acid: a computer simulation. Ther Drug Monit. 2005;27:163-7.

16. Jeong H, Kaplan B. Therapeutic monitoring of mycophenolate mofetil. Clin J Am Soc Nephrol. 2007;2:184-91.

17. Pawinski T, Hale M, Korecka M, Fitzsimmons WE, Shaw LM. Limited sampling strategy for the estimation of mycophenolic acid area under the curve in adult renal transplant patients treated with concomitant tacrolimus. Clin Chem. 2002;48:1497-504.

18. Li P, Shuker N, Hesselink DA, van Schaik RH, Zhang X, van Gelder T. Do Asian renal transplant patients need another mycophenolate mofetil dose compared with Caucasian or African American patients? Transpl Int. 2014:27:994-1004.

19. Kuypers DR, Le Meur Y, Cantarovich M, Tredger MJ, Tett SE, Cattaneo D, et al. Consensus report on therapeutic drug monitoring of mycophenolic acid in solid organ transplantation. Clin J Am Soc Nepohrol. 2010;5:341-58.

20. Miura M, Satoh S, Niioka T, Kagaya H, Saito M, Hayakari M, et al. Limited sampling strategy for simultaneous estimation of the area under the concentration-time curve of tacrolimus and mycophenolic acid in adult renal transplant recipients. Ther Drug Monit. 2008;30:52-9.

21. Miura M, Satoh S, Niioka T, Kagaya H, Saito M, Hayakari M, et al. Early phase limited sampling strategy characterizing tacrolimus and mycophenolic acid pharmacokinetics adapted to the maintenance phase of renal transplant patients. Ther Drug Monit. 2009;31:467-74.

22. Yamaguchi K, Fukuoka N, Kimura S, Watanabe M, Tani K, Tanaka H, et al. Limited sampling strategy for the estimation of mycophenolic acid area under the concentration-time curve treated in Japanese living-related renal transplant recipients with concomitant extended-release tacrolimus. Biol Pharm Bull. 2013;36:1036-9.

23. Bullingham RE, Nicholls A, Hale M. Pharmacokinetics of mycophenolate mofetil (RS61443): a short review. Transplant Proc. 1996:28:925-9. 